\title{
Effects of a preferred vs a nonpreferred CS in the establishment of a taste aversion
}

\author{
FRANK ETSCORN \\ Western Kentucky University, Bowling Green, Ky. 42101
}

\begin{abstract}
Following ingestion of either saccharin-flavored water (a preferred substance) or HCL-flavored water (nonpreferred), two groups of mice were injected intraperitoneally with an illness inducer to establish a conditioned taste aversion. After 2 recovery days, the aforementioned procedure was repeated, followed by 2 more recovery days. An extinction trial was then introduced, which consisted of presenting the taste cues and omitting the injections. Results indicated that the aversion to the HCL was stronger in all cases than it was to the saccharin.
\end{abstract}

Taste aversions in the rat as well as in other animals appear when a distinctly flavored fluid (CS) is paired with an illness (US) induced by such means as drug injection or X-irradiation. Following recuperation, the CS will be avoided if it is once more presented to the animal. Recent studies in the area have indicated that when two or more CSs are used independently of each other in the establishment of a taste aversion, the CS most preferred will generally yield the strongest aversion. Wittlin and Brookshire (1968) reported that a taste aversion was established only to the more palatable of two novel substances. Their design employed the sensitive two-bottle preference method (Dragoin, 1971) for a consumption measure. The CS was either grape juice (least palatable) or milk (most palatable), with the US being apomorphine. Results by Green and Churchill (1970) indicated that taste aversions to a preferred CS were stronger than they were for a nonpreferred CS. These Es used both the one- and two-bottle test, with the CSs being either milk (preferred) or grape juice (nonpreferred). The US was apomorphine. Somewhat inconsistent with these data were the findings of Kalat and Rozin (1970). These researchers reported that when using the two-bottle test, animals ranked the CSs from least to most aversive (on the basis of the established aversion) as vanilla, followed by salt, sucrose, and casein hydrolysate. The US was lithium chloride. Controls ranked the CSs from least to most attractive as casein hydrolysate, vanilla, salt, and sucrose. However, except for casein hydrolysate, the other three substances were still ranked in a manner consistent with the notion that stronger preferences yield stronger aversions. The present study was undertaken in an attempt to determine the strength of a taste aversion for a preferred vs a nonpreferred substance relative to a distilled water baseline.

\section{METHOD}

Eight male albino mice from the Western Kentucky University animal colony, weighing $31-44 \mathrm{~g}$ at the beginning of the experiment, were used as Ss. The Ss had been maintained since weaning on Purina Lab Chow and never been subjected to any liquid substance other than water. Ss were housed individually in plastic cages $\left(7 \frac{1}{4} \times 4 \frac{1}{4} \times 3 \frac{1}{4}\right.$ in. high) with free access to Lab Chow for the duration of the experiment. Temperature was maintained at a constant $75^{\circ} \mathrm{F}$, and a 12 -h day-night cycle was used. All animals were adapted to a drinking schedule consisting of 15-min daily access to distilled water for 7 days. Each $\mathrm{S}$ was assigned two stainless steel drinking tubes and two glass bottles, one set for its distilled water and the other for its taste cue. On Day 8 (Treatment 1), Ss were assigned randomly to either a saccharin CS group (SG) or an HCL CS group (HG). Each $\mathrm{S}$ in the HG was then allowed access to $30 \mathrm{ml}$ of the HCL CS $[.20 \mathrm{cc}$ of standard HCL (28\%) diluted in $200 \mathrm{ml}$ of distilled water]. Likewise, each $\mathrm{S}$ in the $\mathrm{SG}$ was given access to $30 \mathrm{ml}$ of the saccharin CS $(1 / 2$ grain of saccharin diluted in $200 \mathrm{ml}$ distilled water). After $15 \mathrm{~min}$ access, the drinking bottles for both groups were removed and the amount consumed per $S$ was measured and recorded. At this time, the animals were weighed and their US dosages computed. The US for both groups was cyclophosphamide (Cytoxan $\mathbf{R}$, Mead-Johnson Laboratories, Evansville, Indiana) at an intensity of $66 \mathrm{mg} / \mathrm{kg}$ of $\mathrm{S}$ body weight. Thirty minutes following removal of the CS, both groups were injected intraperitoneally with the US. Approximately $30 \mathrm{sec}$ were required to remove an animal from its cage, inject, and return it. Following injections, all Ss were allowed 2 recovery days (Days 9 and 10) with access to $30 \mathrm{ml}$ of distilled water on the normal 15-min schedule. On Day 11 (Treatment 2), the Ss were again presented with the same CSs for $15 \mathrm{~min}$, and the amount consumed per animal was recorded. Following this treatment, the animals were again weighed, their dosages computed, and again injected with the US. Days 12 and 13 were recovery days. Day 14 (Treatment 3 ) consisted of presenting the appropriate CS and measuring consumption. No US was administered. The distilled water during adaptation and the CSs on treatment days were presented at 12:00, with US administration following $45 \mathrm{~min}$ later on the appropriate days.

\section{RESULTS}

Figure 1 represents the amount consumed in ccs for both the HG and SG animals. Consumption measures taken for Treatment 1 established the degree of preference and nonpreference for the two CSs relative to the distilled water. Pilot studies had been used to match the intensities for the two CSs relative to their absolute deviations from their previously established distilled water baseline (mean consumption of distilled water for the last 5 days of adaptation was 3.95; mean consumption for the SG on Treatment 1 was 4.50 ; mean consumption for the HG on Treatment 1 was 3.50). 


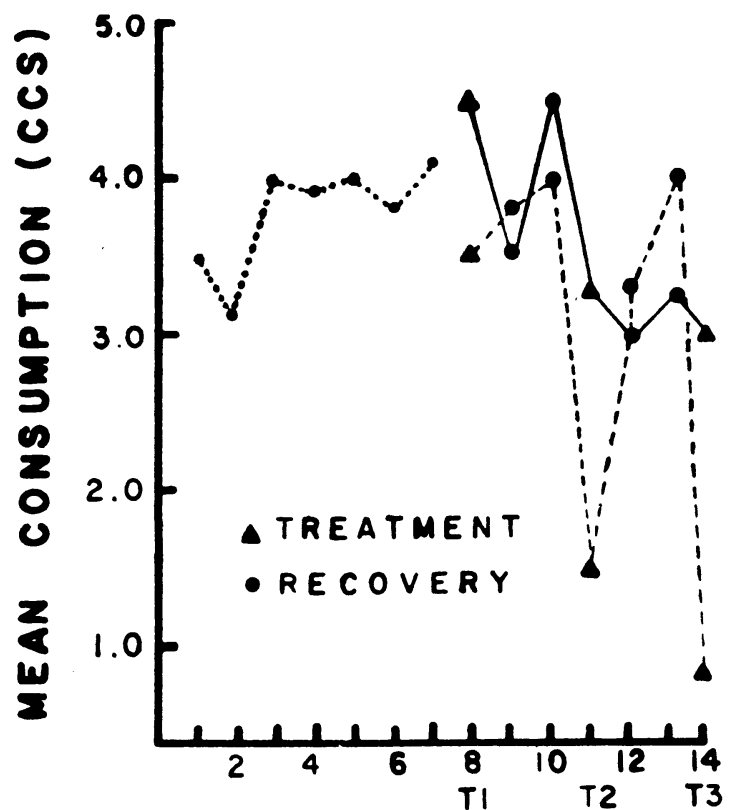

DAYS

Fig. 1. Mean intake of distilled water during adaptation (dotted line) and for the saccharin group (continuous line) and HCL group (broken line).

Comparing fluid intake for Treatment 2 SG with Treatment 2 HG and Treatment 3 SG with Treatment 3 HG indicated that the aversion was stronger in both cases to the HCL (Mann-Whitney U, p $<.014$ for both cases). It can also be seen from Fig. 1 that the HG demonstrated a considerable decrement in consumption from Treatment 1 to Treatment 2 (Walsh, p <.062), while the SG evidenced no significant change.

The results indicate that with the present CSs the aversion was much stronger to the nonpreferred CS than to the preferred CS. In fact, when the extinction schedule was instituted for 3 more days following Treatment 3 , the HG Ss still displayed a strong aversion to the HCL (Walsh, $p<.062$ ). On the other hand, the SG animals returned to their Treatment 1 consumption level following the aforementioned schedule.

\section{DISCUSSION}

While the results of the present experiment appear to differ from the findings of other researchers in the area of taste aversion learning, the possibility exists that the use of the less sensitive one-bottle test could have had an effect on the present findings. Current research suggests that the more sensitive two-bottle test (Dragoin, 1971) is the preferred method in taste aversion studies. Consequently, research is presently under way using the two-bottle test to verify the results reported above.

Another observation concerns the generally accepted notion that taste aversion learning can normally be demonstrated with only one CS-US pairing (assuming, of course, sufficient CS-US intensity and a minimal delay of reinforcement). Although this held true for the HCL group, the aversion was not seen in the saccharin group until Treatment 3.

Finally, it has been suggested that stronger aversions to preferred substances occurred simply because more of the particular CS was consumed, thus giving a "stronger" cue effect. The present experiment did not demonstrate this since more saccharin than HCL was consumed in all presentations; yet, the aversion was stronger to the least consumed substance, HCL.

\section{REFERENCES}

Dragoin, W. B. A comparison of two methods of measuring conditioned taste aversions. Behavioral Research Methods \& Instrumentation, 1971, 3, 309-310.

Green, K. G., \& Churchill, P. A. An effect of flavor on strength of conditioned aversions. Psychonomic Science, 1970, 21, 19-20.

Kalat, J. W., \& Rozin, P. "Salience." A factor which can override temporal contiguity in taste aversion learning. Journal of Comparative \& Physiological Psychology, 1970, 71, 192-197.

Wittlin, W. A., \& Brookshire, K. B. Apomorphine-induced conditioned aversions to a novel food. Psychonomic Science, $1968,12,217-218$.

(Received for publication July 19, 1972; revision received August 14, 1972.) 